\title{
Experimental path loss model for liver implanted wireless communication channel at ultra-wideband range
}

\author{
Pongphan Leelatien ${ }^{1 a)}$, Koichi Ito ${ }^{2}$, and Kazuyuki Saito ${ }^{2}$ \\ ${ }^{1}$ Graduate School of Engineering, Chiba University, \\ 1-33 Yayoi-cho, Inage-ku, Chiba 263-8522, Japan \\ ${ }^{2}$ Center for Frontier Medical Engineering, Chiba University, \\ 1-33 Yayoi-cho, Inage-ku, Chiba 263-8522, Japan
}

a)pongphan@chiba-u.jp

\begin{abstract}
With the potential use of wireless implanted devices for transplanted liver monitoring applications, it is important to investigate the inbody propagation channel at liver area and to demonstrate the feasibility of the applications. Experimental measurements were performed using our developed multilayer human equivalent phantoms. Subsequently, path loss data were recorded and analyzed for various distances between the implanted and on-body antennas at lower range of ultra-wideband to assess the in-body propagation channel. Finally, our preliminary results indicate a possibility for liver implanted wireless communications using UWB technology in the example case scenario.
\end{abstract}

Keywords: ultra-wideband (UWB), liver implanted wireless communication, path loss model, transplanted liver monitoring

Classification: Antennas and Propagation

\section{References}

[1] M. N. Ericson, M. A. Wilson, G. L. Coté, J. S. Baba, W. Xu, M. Bobrek, C. L. Britton, M. S. Hileman, M. R. Moore, M. S. Emery, and R. Lenarduzzi, "Implantable sensor for blood flow monitoring after transplant surgery," Minim. Invasive Ther. Allied Technol., vol. 13, no. 2, pp. 87-94, Apr. 2004. DOI:10. 1080/13645700410027866

[2] M. I. Prince and M. Hudson, "Liver transplantation for chronic liver disease: Advances and controversies in an era of organ shortages," Postgrad. Med. J., vol. 78, no. 917, pp. 135-141, Mar. 2002. DOI:10.1136/pmj.78.917.135

[3] T. J. Akl, M. A. Wilson, M. N. Ericson, E. Farquhar, and G. L. Coté, "Wireless monitoring of liver hemodynamics in vivo," PLoS One, vol. 9, no. 7, p. e102396, Jul. 2014. DOI:10.1371/journal.pone.0102396

[4] A. Khaleghi, R. Chávez-Santiago, and I. Balasingham, "Ultra-wideband statistical propagation channel model for implant sensors in the human chest," IET Microw. Antennas Propag., vol. 5, no. 15, pp. 1805-1812, Dec. 2011. DOI:10.1049/iet-map.2010.0537 
Ramstad, and I. Balasingham, "In-body to on-body ultra wideband propagation model derived from measurements in living animals," IEEE J. Biomed. Health Inform., vol. 19, no. 3, pp. 938-948, May 2015. DOI:10.1109/JBHI.2015. 2417805

[6] J. Shi and J. Wang, "Channel characterization and diversity feasibility for inbody to on-body communication using low-band UWB signals," Proc. 3rd International Symposium on Applied Sciences in Biomedical and Communication Technologies (ISABEL), Roma, Italy, pp. 1-4, Nov. 2010. DOI:10.1109/ ISABEL.2010.5702784

[7] H. Bahrami, S. A. Mirbozorgi, L. A. Rusch, and B. Gosselin, "Biological channel modeling and implantable uwb antenna design for neural recording systems," IEEE Trans. Biomed. Eng., vol. 62, no. 1, pp. 88-98, Jan. 2015. DOI:10.1109/TBME.2014.2339836

[8] P. Leelatien, K. Ito, and K. Saito, "Wireless monitoring of transplanted organ at UWB range," IEICE Society Conference 2016, B-1-82, Sep. 2016.

[9] S. Gabriel, R. W. Lau, and C. Gabriel, "The dielectric properties of biological tissues: II. Measurements in the frequency range $10 \mathrm{~Hz}$ to $20 \mathrm{GHz}$," Phys. Med. Biol., vol. 41, no. 11, pp. 2251-2269, Apr. 1996. DOI:10.1088/0031-9155/41/ $11 / 002$

\section{Introduction}

Recently, wireless implanted device is of an interest in the area of medicine for healthcare monitoring and collecting biological parameters. One of the promising wireless implanted applications is physiological monitoring of transplanted organs. Nowadays, organ transplantation has been continuously performed during medical treatments [1]. Particularly, liver transplantation has become the primary clinical treatment for end stage liver disorders [2]. However, the technical failure rate is reported to be highest during the first two-week period after surgery [3]. Conventional techniques such as periodic blood testing and liver tissue biopsy do not offer real-time and constant monitoring after organ transplantation and are often too slow to respond to the potential loss of transplanted organ. Being one of the most common transplanted solid organs with only a limited number of organ donors, this strongly emphasizes the need to minimize liver transplantation failure [1].
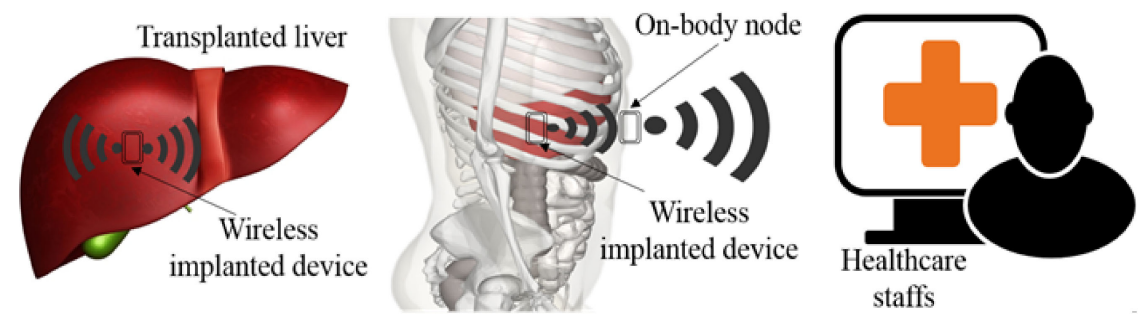

Fig. 1. An overview of the wireless implanted monitoring system.

Fig. 1 demonstrates an example scenario for the wireless implanted monitoring system. Wireless implanted device positioned on the liver surface monitors and transmits body parameters such as blood flow and oxygen saturation which are predictors of transplanted liver function [3] to on-body node which acts as a 
relay for wireless transmissions to personal devices or hospital servers. This application can provide a reliable method of instantaneous and continuous monitoring to report the organ status of a patient to any healthcare staffs. This early detection should enable prompt medical operations before serious damage occurs to the transplanted liver $[1,3]$. Moreover, together with future biodegradable materials, an implanted device will not need to be removed from a patient, thus, patient risks will be reduced. Eventually, this will potentially improve the success rate of liver transplantation.

On the other hand, it is important to select the appropriate frequency band of wireless communications between the devices that is suitable to the specific applications in consideration. Ultra-wideband (UWB) technology is a favorable option for wireless implanted applications [4]. Due to its high frequency range, the physical size of implantable antennas can be significantly reduced. Moreover, since it has a simplicity on the transmitter side, this will lead to the miniaturization of implanted devices. Its low power consumption will extend the implanted device longevity. However, at UWB range, the signals encounter severe loss inside the human body leading to the difficulty of system implementation. Therefore, it is important to evaluate the characteristics of propagation channel at UWB range to achieve reliable in-body wireless communication links. There have been various attempts to study UWB propagation channel at various locations inside human body e.g. chest [4], abdomen [5, 6], and brain [7]. Nevertheless, to the authors' knowledge, UWB channel characteristics at liver location for liver implanted wireless monitoring system has not been reported in any open literature. Hence, to this aim, we performed experimental measurements using our developed simplified multilayer human equivalent phantoms to obtain attenuation data and proposed path loss model which would provide initial understandings of the characteristics of liver implanted wireless communications using UWB channel [8].

\section{UWB antenna and measurement setup}

The antennas used as the on- and in-body antennas in our measurements were Skycross UWB antennas (SMT-3TO10M-A) manufactured by Skycross Inc. as shown in Fig. 2(a). Experimental measurements were conducted using multilayer phantoms as illustrated in Figs. 2(b) and 2(c). The measurement setup consisted of the two Skycross UWB antennas (SMT-3TO10M-A) as shown in Fig. 2(a), an Agilent N5230C PNA-L vector network analyzer (VNA), two coaxial cables connecting each antenna to port 1 and port 2 of the VNA, and the human equivalent semi-solid UWB phantoms of fat, muscle, and liver tissues. The whole size of phantoms was approximately $200 \mathrm{~mm} \times 130 \mathrm{~mm} \times 120 \mathrm{~mm}$. The cables' frequency response was subtracted from the channel measurements by the method of VNA calibration. A broadband measurement of phantom dielectric properties was conducted to confirm their values compared to values reported in the work of Gabriel et al. [9]

$S_{21}$ data within the frequency range of 3-5 GHz for various distances between the antennas in the measurements were recorded five times and averaged. Our measurements were set up as followed. One antenna located on the liver surface 
under the muscle layer (referred to as in-body antenna) was at a fixed position while the other antenna (referred to as on-body antenna) was placed directly on the fat layer and moved from the beginning point $(0 \mathrm{~mm})$ in steps of $20 \mathrm{~mm}$ in each measurement up to the ending point $(100 \mathrm{~mm})$. Both antennas were placed in a face-to-face orientation as presented in Fig. 2(b).

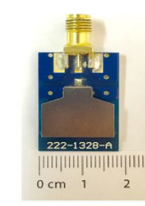

a) Skycross UWB antenna (SMT-3TO10M-A) used as the on- and the in-body antennas in the measurements

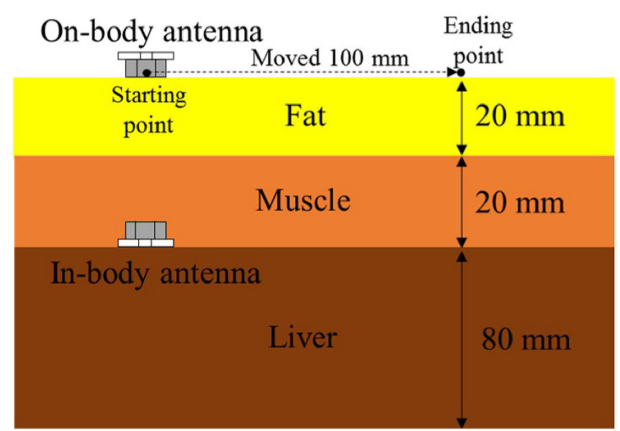

b) Cross-sectional view of measurement setup with the locations and the orientations of the onand the in-body antennas

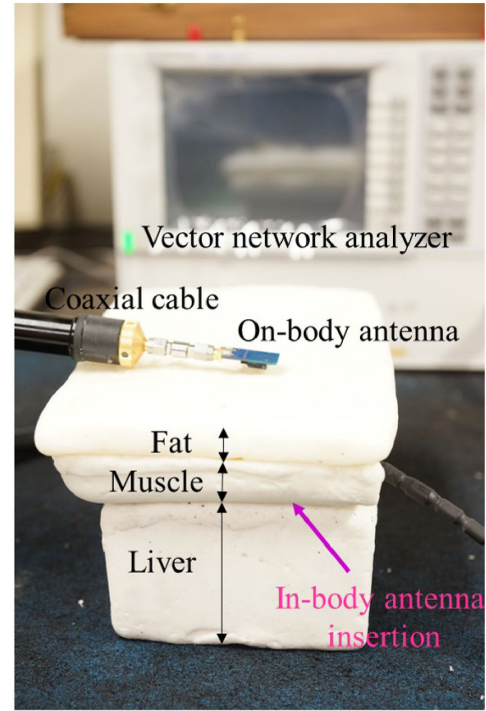

c) Measurement setup with the onbody antenna, the in-body antenna, and the multilayer phantoms consisted of fat, muscle, and liver layers

Fig. 2. Measurement setup.

\section{Results}

Firstly, to confirm the performance of the antennas, we recorded the $S_{11}$ result of the on-body antenna and the $S_{22}$ result of the in-body antenna within the $3-5 \mathrm{GHz}$ range as illustrated in Figs. 3(a) and 3(b) respectively. We can see that within the considered frequency range, the on-body antenna shows good behavior. On the other hand, since this UWB antenna was originally designed for free space utilization, the performance of the in-body antenna is not good but is acceptable for our research purpose here. Subsequently, with the in-body antenna at the fixed position, we moved the on-body antenna from the starting point $(0 \mathrm{~mm})$ to the ending point $(100 \mathrm{~mm})$ and obtained experimental results of $S_{21}$ parameter for various separation distances between the in- and on-body antennas. Then, to evaluate the signal attenuations inside the human body, we later processed all recorded $S_{21}$ data from the measurements to obtain the path loss (PL) data of each link as PL $=-\operatorname{mean}\left\{\left|S_{21}\right|\right\}$ in decibels. Consequently, we obtained the path loss model of the in-body channel as a function of the propagation distance by applying linear fitting to the path loss data at all points. The log-distance path loss model was applied as the following expression: 


$$
\mathrm{PL}_{\mathrm{dB}}(d)=\mathrm{PL}_{0, \mathrm{~dB}}+10 n \log _{10}\left(\frac{\boldsymbol{d}}{\boldsymbol{d}_{0}}\right)
$$

where $d$ is the separation distance in millimeters between the in- and on-body antennas and $d_{0}$ is the reference depth of implantation which is $40 \mathrm{~mm} . \mathrm{PL}_{0}$ is the path loss at the reference location, $d_{0}=40 \mathrm{~mm}$. The exponent, $n$, which is known as path loss exponent, can be used to evaluate how fast the signal power attenuates with the distance. Fig. 3(c) shows the average path loss data collected from the measurements as a function of the logarithmic antenna separation distances and the linear fitting curve of the measured data. In our case, $\mathrm{PL}_{0}$ was $47.95 \mathrm{~dB}$. The path loss exponent which is the slope of the obtained fitting curve was 6.25 .

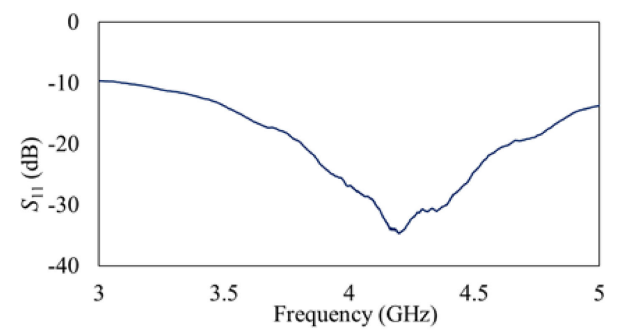

(a) The reflection coefficient of the on-body antenna

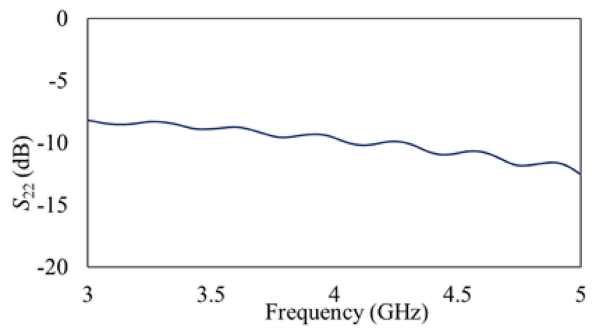

(b) The reflection coefficient of the in-body antenna

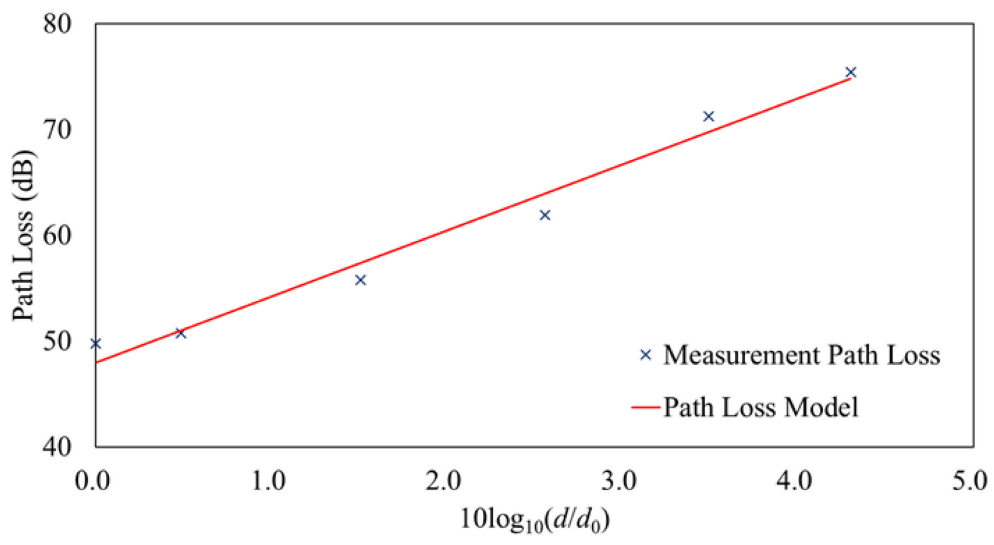

(c) Measured path loss data for in-body channel as a function of the logarithmic inand on-body antenna separation distance

Fig. 3. Measured results and path loss model.

\section{Conclusion}

The propagation characteristics of in-body channels using UWB technology for liver implanted wireless communications have been investigated for the first time 
by mean of experimental measurements using simplified multilayer human equivalent phantoms. This study provides important preliminary insights for liver implanted wireless monitoring system. The in-body path loss data for various propagation distance values between the implanted and on-body antennas within the 3-5 GHz band obtained using measurement approaches were presented. It was found that path loss data were between $45 \mathrm{~dB}$ and $80 \mathrm{~dB}$ within $40 \mathrm{~mm}$ and $100 \mathrm{~mm}$ propagation range approximately. Our preliminary studies in this paper show that the realization of liver implanted wireless monitoring system using UWB channel in the example scenario case is possible. Moreover, our results reveal path loss data for liver-skin surface communication channel which is a necessary parameter for the design and evaluation of liver implanted wireless communication links. The information here can be used as guidelines for future studies of various in vivo medical applications such as wireless implanted monitoring of organs and implantable drug delivery at precise targeted locations and would also encourage other innovative applications utilizing UWB technology.

For our future work, due to the necessity to accurately characterize the in-body wireless communication channel at liver location and to further confirm the feasibility of liver implanted wireless monitoring system in the example scenario case, we will continue our further investigations using antennas designed for the implantation at liver location. In addition, we will conduct simulations using various digital human models as well as experimental measurements using realistic human phantoms including liver and other internal organs around the liver area to obtain insights of antenna performances and propagation channels for liver implanted wireless communications under more realistic environments.

\section{Acknowledgments}

The authors would like to thank Dr. Akram Alomainy, Queen Mary University of London, for the useful discussions on the in-body wireless communications during this research and AET, Inc., Japan for their supports on CST Microwave Studio. 